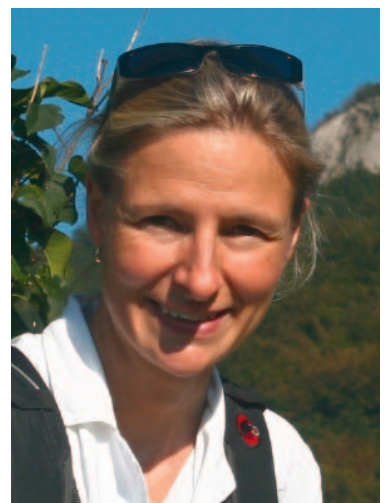

Frau Prof. Glasl-Tazreiter, Sie sind am Department für Pharmakognosie der Universität Wien tätig und beschäftigen sich vornehmlich mit Ethnopharmakognosie, Pharmakobotanik und Phytochemie. Können Sie uns die jeweiligen Aufgabengebiete und Ihre gegenwärtigen Tätigkeiten beschreiben?

Die Ethnopharmakognosie beschäftigt sich mit dem «Wissen vom Heilen» in verschiedenen traditionellen Medizinalsystemen. Dabei sind die Art der Erkrankungen im entsprechenden Kulturkreis sowie die Arzneien und deren Zubereitungsformen, die zur Heilung verwendet werden, von Interesse. Der Fokus liegt klar auf den verwendeten Heilpflanzen, die neben anderen biologischen Materialien in den Rezepturen verarbeitet werden. Die Ethnopharmakognosie stellt ein interdisziplinäres und interkulturelles Forschungsgebiet dar, das die Zusammenarbeit von Medizin, Pharmazie, Ethnologie und Botanik erfordert. Jene Bereiche, in denen das Department für Pharmakognosie der Universität Wien Beiträge liefern kann, liegen vor allem auf dem Gebiet der Pharmakobotanik und Phytochemie.

Ist es gelungen, durch Zusammenarbeit mit Kräuterkundigen in einem Kulturkreis eine Arzneipflanze aufzuspüren, die bei einer bestimmten

\title{
Ethnopharmakognosie und Ganzheitsmedizin
}

Ao. Univ.-Prof. Dr. Sabine Glasl-Tazreiter arbeitet und doziert am Department für Pharmakognosie der Universität Wien, Österreich. Zudem ist sie als Generalsekretärin der Österreichischen Pharmazeutischen Gesellschaft (OePhG) und als Gutachterin für Zeitschriften wie Planta Medica, Phytochemistry und Scientia Pharmaceutica aktiv. In der SchweIzeRISCHEN ZeITSCHRIFT FÜR GANZHEITSMEDIZIN hat Frau Prof. Glasl-Tazreiter unter anderem Erkenntnisse zum Thema Hoodia publiziert (2009;21:300-306).

Indikation verwendet wird, beginnt die Arbeit des Pharmakobotanikers. Seine Aufgabe besteht darin, die Identität der Pflanze zweifelsfrei zu bestimmen und somit die so wichtige Grundlage für jegliche künftige Forschungsarbeit zu schaffen. Die Botanik stellt sozusagen das Fundament für die weitere wissenschaftliche Arbeit an der betreffenden Pflanze dar. Diese Tatsache erscheint mir von grösster Wichtigkeit, da ihr leider manchmal selbst in Fachkreisen zu wenig Bedeutung beigemessen wird. Gelingt es nicht, das Material botanisch korrekt zuzuordnen, so sind alle weiteren Ergebnisse wertlos und somit nicht publizierbar, da man keine Aussage darüber treffen kann, für welche Stammpflanze die erhobenen Daten Gültigkeit besitzen. Die Arbeit des Pharmakobotanikers ist manchmal nicht ganz einfach. Als Voraussetzung werden korrekt angefertigte Herbarbelege benötigt, die jedoch nicht immer zur Verfügung stehen. Ferner ist nicht immer die erforderliche Literatur mit ausführlichen makroskopischen und mikroskopischen Beschreibungen zur Hand, obwohl das Organisieren entsprechender Literatur durch das Internet im Vergleich $\mathrm{zu}$ früher wesentlich erleichtert ist. Manchmal benötigt man neben Blättern und Blüten auch andere Organe, Samen oder Früchte, die aber zur Zeit der Aufsammlung nicht verfügbar waren. Hier bewähren sich gute und verlässliche Kontakte in die Region, um im Nachhinein fehlendes pflanzliches Material für die Bestimmung zu beschaffen.

Sobald die Identität der Stammpflanze geklärt ist, beginnt der Phytochemiker mit der Extraktion, die sich idealerweise an der Zubereitungsform in der traditionellen Medizin orientiert (Bereitung von Infus, Dekokt, alkoholischer Zubereitung usw.). Das Extrakt wird durch FlüssigFlüssig-Verteilung gereinigt und anhand verschiedener chromatographischer Techniken in viele Fraktionen aufgetrennt. Um Kenntnis darüber zu erlangen, welche dieser Fraktionen wirksame Inhaltsstoffe enthält, braucht der Phytochemiker Testsysteme, in die die Fraktionen eingeschleust und hinsichtlich ihrer Wirksamkeit überprüft werden. Diese Vorgehensweise wird als «aktivitätsgeleitete Fraktionierung» bezeichnet und ermöglicht das Aufspüren potenzieller Wirkstoffe. Ziel ist es, Reinsubstanzen $\mathrm{zu}$ isolieren und deren Struktur zu klären. Methoden wie die Massenspektrometrie, UV- und IRSpektroskopie sowie die Kernresonanzspektroskopie liefern strukturelle Informationen, die der Phytochemiker wie Teile eines Puzzles zusammensetzt, um die dreidimen- 
sionale Struktur einer neu isolierten Verbindung zu beschreiben. Häufig beobachtet man, dass eine Fraktion, die in den Tests zunächst eine sehr gute Wirksamkeit gezeigt hat, durch zunehmende Aufreinigung ihre Aktivität wieder verliert. Somit ist es oftmals unmöglich, die Wirksamkeit auf nur eine einzige Substanz zurückzuführen, was aber nicht heisst, dass die Arzneipflanze unwirksam ist. Ganz im Gegenteil: Die Aktivität kommt erst durch die synergistischen Wirkungen vieler verschiedener Einzelkomponenten zustande, die jedoch wieder verloren geht, wenn weiter aufgetrennt wird. In diesen Fällen stellt der Gesamtextrakt oder die angereicherte Fraktion in ihrer komplexen Zusammensetzung den "Wirkstoff» dar. Es ist daher von grosser Bedeutung, diesen Wirkstoffkomplex möglichst gut $\mathrm{zu}$ charakterisieren. Der Phytochemiker ist gefordert, analytische Systeme zu entwickeln, um eine qualitative und quantitative Bestimmung der isolierten Komponenten zu ermöglichen. Das gewährleistet darüber hinaus den Vergleich von Pflanzen unterschiedlicher Herkunft (Standort, Erntejahr, Erntezeitpunkt) sowie die Überprüfung verschiedener Extraktchargen.

Sie haben sich im Zusammenhang mit phytotherapeutischen Arzneimitteln, $z . B$. traditionellen Heilmitteln von indigenen Bevölkerungen, unter anderem mit den Aspekten Patentierung sowie geistiges Eigentum befasst. Was können Sie uns hierzu berichten?

Im Rahmen des «Erdgipfels», der Konferenz der Vereinten Nationen über Umwelt und Entwicklung, die vom 3. bis 14. Juni 1992 im brasilianischen Rio de Janeiro stattfand, wurde die Biodiversitätskonvention implementiert. Die «Convention on Biological Diversity» (CBD) hat drei gleichrangige Ziele:
- Schutz der biologischen Vielfalt.

- Nachhaltige Nutzung ihrer Bestandteile.

- Zugangsregelung und gerechter Ausgleich von Vorteilen, welche aus der Nutzung genetischer Ressourcen entstehen (Access and Benefit Sharing, ABS).

Die Europäische Union hat die CBD unterzeichnet, und das verpflichtet uns, bei unseren Forschungsarbeiten diese drei Ziele zu berücksichtigen. Indigenes Wissen stellt geistiges Eigentum dar; sollte dessen Nutzung z.B. zur Entwicklung eines vielversprechenden Wirkstoffs führen, so müssen im Falle eines Profits Tantiemen an die Ethnie zurückfliessen. Beforscht man Arzneipflanzen aus fremden Kulturen und resultieren daraus publizierbare Ergebnisse, so verlangen hochkarätige Journale heutzutage einen Nachweis über die ordnungsgemässe Bewilligung der Ausfuhr aus dem entsprechenden Land. Damit soll der «Biopiraterie» Einhalt geboten werden. In den Projekten an unserem Department existiert meist eine enge Kooperation mit Kollegen vor Ort. Das erleichtert die gesamten Abläufe in der Zusammenarbeit durch das Zurückgreifen auf ein vorhandenes lokales Netzwerk. Zumeist ermöglicht es auch eine bessere Kommunikation mit den Vertretern der indigenen Bevölkerung. Die Ethnopharmakognosie erfordert es zu lernen, «dieselbe Sprache zu sprechen». Vor allem im Hinblick auf Linguistik, wobei Kollegen vor Ort von grösster Bedeutung sind, die sowohl die indigene als auch die englische Sprache beherrschen. Darüber hinaus sieht man sich häufig einem völlig anderen Medizinsystem gegenüber, mit anderem Zugang zu Erkrankungen und deren Heilung. Hier sind Kollegen, die sowohl diese Medizinsysteme als auch das westliche kennen, von grösstem Wert. Im Idealfall gelingt es, Fachleute des jeweiligen Landes und Vertreter der Indigenen in das Projekt einzubinden, um eine
«Win-Win-Situation» mit Vorteilen für beide Seiten zu gewährleisten.

Welche «Neuentdeckungen» traditioneller Heilmittel haben in den letzten Jahren Eingang in das Repertoire der westlichen Medizin gefunden? Inwiefern schätzen Sie deren Einsatz in Schul-und Komplementärmedizin ein?

Die Wurzeln von Pelargonium sidoides wurden im südafrikanischen Raum bei Erkältungskrankheiten und Erkrankungen der Lunge, z.B. durch Siedler eingeschleppte Tuberkulose, eingesetzt. Für die Wurzelextrakte konnte eine immunmodulatorische Wirkung belegt werden, die allerdings keiner distinkten chemischen Stoffgruppe zuzuschreiben ist. Ferner wirken die Extrakte antibakteriell und zeigen Wirksamkeit gegen einige Mykobakterienstämme. Das in Österreich als traditionelles pflanzliches Arzneimittel registrierte $\mathrm{Kaloba}^{\circledR}$ wird gerne empfohlen - vermehrt von Kinderärzten, die damit in der Prophylaxe und Therapie wiederkehrender Atemwegsinfekte in der kalten Jahreszeit sehr gute Erfahrungen gemacht haben.

Katzenkrallenwurzel stammt aus der traditionellen Medizin der im zentralperuanischen Regenwald beheimateten Asháninka-Indianer. Uncaria tomentosa findet Einsatz bei Erkrankungen des rheumatischen Formenkreises, bei viralen und bakteriellen Infektionen sowie Tumor- und allergischen Erkrankungen. Das Wissen über diesen Arzneischatz gelangte Mitte der Siebzigerjahre nach Europa und fand Eingang in unsere westliche Medizin. In Österreich ist seit dem Jahr 2000 das Präparat Krallendorn ${ }^{\circledR}$ _ Kapseln, ein standardisierter Trockenextrakt aus $U$. tomentosa, als Arzneimittel zugelassen. Es kommt zur Zusatzbehandlung bei einer antirheumatischen Basistherapie und im Bedarfsfall einer Schmerztherapie bei Patienten mit rheumatoider Arthritis 
zum Einsatz. Darüber hinaus wurden für Extrakte aus Katzenkrallenwurzel immunstimulierende, antivirale, antioxidative und vasodilatatorische Effekte nachgewiesen.

Der Strauch bzw. kleine Baum Morinda citrifolia stammt aus Südostasien (Polynesien, Hawaii, Australien). Seine Wurzel, die Stammrinde und die Blätter werden in der traditionellen Medizin bei Bluthochdruck, Diabetes, Durchfall, Infektionen, Malaria und Schmerz verwendet. Bei uns erfreuen sich die Früchte von Morinda in Form des Noni-Saftes grösster Beliebtheit. Während die Früchte in der traditionellen Medizin als Nahrungsmittel in Hungersnöten bekannt sind, werden sie in unseren Breiten als Allheilmittel propagiert (Entzündungshemmung, Leber-, Milz-, Nierenerkrankungen, Bluthochdruck, Diabetes, Übergewicht, Schlafstörungen, Kopfschmerz, Zahnschmerz, Depression, Krebs und viele mehr). Mit der Belegung der Wirksamkeit bei all diesen Indikationen schaut es jedoch sehr mager aus, sodass der Eingang in die westliche Medizin im Sinne eines Phytotherapeutikums nicht zu erwarten ist.

Mit welchen traditionellen Heilmitteln haben Sie bei welchen Erkrankungen/ Anwendungen die besten Erfahrungen gemacht? Welche werden den Weg in die Produktion von Fertigarzneimitteln finden?

Der Wermut zeigt immer wieder eindrucksvoll sein hohes Potenzial bei Übelkeit, Erbrechen, Magenkrämpfen und ähnlichen Symptomen. Es ist jene Teedroge, die bei meiner Grossmutter als Hausmittel der ersten Wahl galt, um «den Magen wieder zu richten». Auch wenn es mich persönlich immer eine riesige Überwindung kostet, eine Tasse des bitteren Getränks zu mir zu nehmen, so halte ich grosse Stücke auf den Wermut, denn er hat bisher ausnahmslos mir selbst und allen «Pro- banden» mit Magen-Darm-Erkrankungen, denen ich diese Droge empfohlen habe, geholfen.

Selbst gesammelte Arnika lindert Kreuzschmerzen in Form von alkoholischen Zubereitungen und heilt Wunden aufgrund ihrer entzündungshemmenden Wirkung. Sie steht auf der «Hitliste» der in der österreichischen Volksmedizin am häufigsten verwendeten Arzneipflanzen ganz oben. Eine Reihe von Fertigarzneimitteln ist in verschiedensten Zubereitungsformen auf dem Markt.

Der Beinwell hilft bei Quetschungen, Verstauchungen, Prellungen, Zerrungen, Blutergüssen und schlecht heilenden Wunden. Er wird volksmedizinisch sehr häufig verwendet, was allerdings auf die externe Anwendung beschränkt bleiben sollte. Zur Behandlung schlecht heilender Wunden rate ich stets zum Pyrrolizidinfreien Fertigarzneimittel; liegt keine offene Wunde vor, so zeigen Umschläge mit der Frischpflanze oder der getrockneten Ware gute Wirkung.

\section{Welches Heilmittel fasziniert Sie} persönlich am meisten? Warum?

Es gäbe einige Beispiele zu nennen; eines der faszinierendsten finde ich das Mutterkorn, Secale cornutum. Der Pilz Claviceps purpurea parasitiert auf Süssgräsern. Vornehmlich wächst er auf Roggen unter Ausbildung von kornähnlichen Dauerformen, die als schwarz-violette Sklerotien aus dessen Ähren herauswachsen. Diese sogenannten Mutterkörner enthalten ein Gemisch an hochwirksamen Alkaloiden (Mutterkorn- oder Ergotalkaloide) mit sehr unterschiedlichen pharmakologischen Wirkungen. Sie waren die Ursache für seuchenartig auftretende Vergiftungen im Mittelalter durch kontaminiertes Brot, das aus befallenem Roggen hergestellt wurde. Die am sogenannten Antoniusfeuer Erkrankten suchten Heilung bei den Antonitern, einem mittelalterlichen Spitalorden, der sich ausschliesslich derjenigen annahm, die am Antoniusfeuer litten oder aufgrund der Krankheit zu Krüppeln geworden waren. Die Antoniter vergaben Auftragswerke an bekannte Maler wie Matthias Grünewald, Martin Schongauer, Niklaus Manuel oder Hieronymus Bosch. So fanden die zwei verschiedenen Verlaufsformen der Vergiftung, Ergotismus gangraenosus und Ergotismus convulsivus, in Form von Höllenszenen und diabolischen Darstellungen Eingang in die bildende Kunst. Die Intention der Antoniter war es, den Kranken diese Werke als bildhafte Predigten zu vergegenwärtigen, die den Betrachter in der Krankheitsbewältigung unterstützen und zum «Heil» führen sollten.

Das Mutterkorn enthält eine Mischung aus verschiedenen Alkaloiden mit komplexem Wirkstoffspektrum, das darauf zurückzuführen ist, dass diese Stoffe als partielle Agonisten bzw. partielle Antagonisten an $\alpha$-adrenergen, Dopamin- und SerotoninRezeptoren wirken. 1918 isolierte Professor Arthur Stoll aus dem Mutterkorn das Peptidalkaloid Ergotamin, das eine uterusaktive Wirkung (Dauerkontraktion), einen vasokonstriktorischen Effekt (Blutdrucksteigerung) sowie eine zentral dämpfende Wirkung besitzt. In den Dreissigerjahren gelang die Isolierung von Ergobasin (= Ergometrin) aus wässrigen Extrakten, das zur Kontraktion der glatten Muskulatur des Uterus und der Gefässe führt. Ab 1935 forschte der Naturstoffchemiker Dr. Albert Hofmann an den Mutterkornalkaloiden und entwickelte Derivate daraus, was schliesslich zur Etablierung einer Reihe von Wirkstoffen führte, die bei Migräne, Hyperprolaktinämie, Morbus Parkinson, Hirnleistungsstörungen, Bluthochdruck und postpartal in der Geburtshilfe angewendet werden. Dies ist ein faszinierendes Beispiel für den grossen Einfallsreichtum der Natur und dafür, welche Vielfalt 
an Wirkungen Substanzen besitzen können, die biogenen Ursprungs sind.

Wie sind Sie mit der Pharmakognosie in Berührung gekommen? Was bedeutet diese für Sie persönlich? Was fasziniert Sie daran besonders?

Mein erster Kontakt mit der Pharmakognosie entstand während des Pharmaziestudiums im Rahmen der phy- tochemischen Übungen. Sowohl die Chemie als auch die Botanik haben mich immer fasziniert, und in der Phytochemie erschien es mir möglich, beides «unter einen Hut zu bringen». Darüber hinaus waren bzw. sind Persönlichkeiten am Wiener Institut, die es verstanden haben, mich für dieses Fach zu begeistern und mir immer wieder neue Blickwinkel zu öffnen. Natürlich sind die Dinge im Fluss, und wie in jeder wissenschaftlichen
Disziplin erfordern neue Erkenntnisse Flexibilität. Genau das ist das Spannende: sich neuen Herausforderungen zu stellen, neu eingeführte Methoden zu erlernen und für sich zu nutzen, Projektpläne zu schmieden und diese umzusetzen.

Sehr geehrte Frau Professor GlaslTazreiter, herzlichen Dank für das Interview!

Interview: Alexander Eitner 